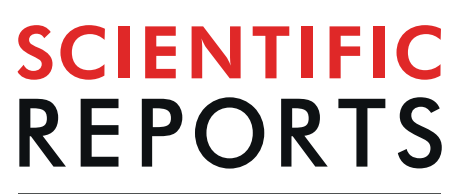

natureresearch

\title{
OPEN Complex nonlinear capacitance in outer hair cell macro-patches: effects of membrane tension
}

\begin{abstract}
Joseph Santos-Sacchi ${ }^{1,2,3^{*}} \&$ Winston $\operatorname{Tan}^{1}$
Outer hair cell $(\mathrm{OHC})$ nonlinear capacitance $(\mathrm{NLC})$ represents voltage sensor charge movements of prestin (SLC26a5), the protein responsible for OHC electromotility. Previous measures of NLC frequency response have employed methods which did not assess the influence of dielectric loss (sensor charge movements out of phase with voltage) that may occur, and such loss conceivably may influence prestin's frequency dependent activity. Here we evaluate prestin's complex capacitance out to $30 \mathrm{kHz}$ and find that prestin's frequency response determined using this approach coincides with all previous estimates. We also show that membrane tension has no effect on prestin's frequency response, despite substantial shifts in its voltage operating range, indicating that prestin transition rate alterations do not account for the shifts. The magnitude roll-off of prestin activity across frequency surpasses the reductions of NLC caused by salicylate treatments that are known to abolish cochlear amplification. Such roll-off likely limits the effectiveness of prestin in contributing to cochlear amplification at the very high acoustic frequencies processed by some mammals.
\end{abstract}

Prestin (SLC26a5) underlies outer hair cell (OHC) mechanical activity ${ }^{1}$, whereby voltage-dependent conformational transitions couple into length changes of the cell (electromotility; $\mathrm{eM})^{2-4}$. Sensor charge movements associated with these conformational changes are measurable as an electrical correlate of eM, i.e., nonlinear capacitance $(\mathrm{NLC})^{5-7}$, which is maximal at $\mathrm{V}_{\mathrm{h}}$, the voltage where prestin charge is distributed equally across the OHC membrane and where eM gain is greatest.

The study of OHC NLC by admittance techniques in whole cell voltage clamp is compromised by contributions from stray capacitance, membrane conductances, and electrode series resistance $\left(\mathrm{R}_{\mathrm{s}}\right)$, the latter altering over time due to plugging of the patch pipette tip with intracellular constituents. Because of this, it is virtually impossible to measure complex membrane capacitance in whole cell mode. We avoid these issues by measuring membrane admittance from macro-patches of the OHC lateral membrane. The lateral membrane of the cylindrical OHC is dominantly populated by prestin $\left(>8000\right.$ functional units $\left./ \mu \mathrm{m}^{2,8-10}\right)$, whereas voltage-dependent membrane conductances are housed in the basal pole membrane ${ }^{11}$. Consequently, macro-patch admittance, following removal of stray capacitance by subtraction of admittance at very depolarized levels where NLC is absent ${ }^{12}$, can be used to study complex sensor-charge movements arising from voltage-induced conformational changes in prestin.

Here, we evaluate patch admittance to provide estimates of complex capacitance representing charge movements both in phase and $90^{\circ}$ out of phase with $\mathrm{AC}$ voltage excitation. We compare such data to previously obtained measures of OHC NLC frequency respons $\mathrm{e}^{13,14}$, and additionally report on the effects of membrane ten$\operatorname{sion}^{15-17}$ on the frequency response of complex NLC. Will an assessment of NLC based on measures of complex NLC alter our current view, as suggested ${ }^{18}$, and can turgor pressure, normally present in the $\mathrm{OHC}^{19}$, with its effect on membrane tension be influential? Our observations indicate that the bulk of sensor charge movement is in phase with voltage, while the resistive component (dielectric loss) is relatively small. Furthermore, membrane tension, though altering prestin's operating voltage point, has no effect on its frequency response. Thus, magnitude estimates of complex NLC are comparable to those measured with other methods ${ }^{13,14}$, being unusually low pass in nature (non-Lorentzian) and indicating that the absolute movement of prestin charge that drives electromotility $(\mathrm{eM})^{20,21}$ is unlikely to extend with high fidelity to the very high acoustic frequencies $(60-160 \mathrm{kHz})$ detected by some mammals.

\footnotetext{
${ }^{1}$ Surgery (Otolaryngology), Yale University School of Medicine, 333 Cedar Street, New Haven, CT, 06510, USA. ${ }^{2}$ Neuroscience, Yale University School of Medicine, 333 Cedar Street, New Haven, CT, 06510, USA. ${ }^{3}$ Cellular and Molecular Physiology, Yale University School of Medicine, 333 Cedar Street, New Haven, CT, 06510, USA. *email: joseph.santos-sacchi@yale.edu
} 


\section{Methods}

All experimental protocols were approved by the Yale Animal Care and Use Committee, and were in accordance with relevant guidelines and regulations. Methods, including details of our voltage chirp stimulus protocol, are detailed in ${ }^{13}$. Briefly, extracellular solution was (in mM): $\mathrm{NaCl} 100$, TEA-Cl 20, $\mathrm{CsCl}$ 20, $\mathrm{CoCl}_{2} 2, \mathrm{MgCl}_{2} 1, \mathrm{CaCl}_{2}$ 1, Hepes 10, pH 7.2. Experiments were performed at room temperature. Extracellular solution was in the patch pipette. On-cell macro-patches on the guinea pig OHC lateral membrane were made near the middle of the cylindrical cell, since prestin density/activity is uniform within the lateral membrane ${ }^{8,22}$. We have previously shown that capacitance frequency response in the $\mathrm{OHC}$ patch remains the same after excision of the patch, indicating that on-cell recording is applicable for our purposes ${ }^{13}$. Borosilicate pipettes of inner tip dimeters between 3-4 $\mu \mathrm{m}$ were used, with M-coat applied within about $20 \mu \mathrm{m}$ of the tip to minimize pipette capacitance. In order to establish gigohm seals we supplemented extracellular solution with $5 \mu \mathrm{M} \mathrm{Gd}^{3+}$; we have shown previously that theses low concentrations help to form seals without affecting $\mathrm{NLC}^{13,23}$. In 2 on-cell patches, we have omitted $\mathrm{Gd}^{3+}$ in the pipette solution and NLC frequency response is not significantly different than in its presence. An Axon 200B amplifier was used with jClamp software (www.scisoftco.com). An Axon Digidata 1440 was used for digitizing at $10 \mu \mathrm{s}$ (Nyquist frequency of $50 \mathrm{kHz}$ ), with a 4-pole Bessel filter of $10 \mathrm{kHz}$. Membrane admittance was determined using a series of voltage chirps ( 4096 points each, resolution $24.4 \mathrm{~Hz}$ ) superimposed onto holding potentials ranging from -160 to $160 \mathrm{mV}$, in $40 \mathrm{mV}$ increments. $100 \mathrm{~ms}$ after each step to a new holding potential, 26 contiguous chirp-induced current responses at each holding potential were time-averaged. Real and imaginary components of the membrane admittance at all chirp frequencies were determined by FFT in jClamp, and corrected for the roll-off of recording system admittance ${ }^{13,24}$. This correction controls for all attenuation and phase characteristics of the system, including the filter, as we have shown previously ${ }^{13}$, providing valid estimates of capacitance beyond the filter cut-off. Our dual-sine methodology permitted us to measure capacitance with high fidelity out to $20 \mathrm{kHz}$ across holding potentials ${ }^{13}$. Here using single sine analysis we extend that to $30 \mathrm{kHz}$.

Our patch pipette inner tip diameters (see Results) were used to estimate the linear capacitance of membrane patches. We estimate a linear membrane patch capacitance of $187.8+/-15.4 \mathrm{fF}(\mathrm{n}=25)$. This was determined by estimating membrane patch hemispheric surface area using the standard value of $1 \mu \mathrm{F} / \mathrm{cm}^{225}$. In our presentation below, we provide absolute estimates of NLC, and specific estimates of NLC by dividing patch admittance with linear capacitance for each patch, thereby accounting for different patch size. Patch experiment data were accepted for inclusion if maximum NLC within our recording bandwidth was $>150 \mathrm{fF}$. Complex values were first averaged for all average-based analyses. Plot traces are smoothed with a 6 point $(150 \mathrm{~Hz}$ bandwidth $)$ running average in Matlab.

In order to extract Boltzmann parameters, capacitance-voltage data were fit to the first derivative of a two-state Boltzmann function.

$$
C_{m}=N L C+C_{s a}+C_{\operatorname{lin}}=Q_{\max } \frac{\mathrm{ze}}{k_{B} T} \frac{b}{(1+b)^{2}}+C_{s a}+C_{\text {lin }}
$$

where

$$
b=\exp \left(-z e \frac{V_{m}-V_{h}}{k_{B} T}\right), \quad C_{s a}=\frac{\Delta C_{s a}}{\left(1+b^{-1}\right)}
$$

$\mathrm{Q}_{\max }$ is the maximum nonlinear charge moved, $\mathrm{V}_{\mathrm{h}}$ is voltage at peak capacitance or equivalently, at half-maximum charge transfer, $\mathrm{V}_{\mathrm{m}}$ is $\mathrm{R}_{\mathrm{s}}$-corrected membrane potential, $z$ is valence, $\mathrm{C}_{\text {lin }}$ is linear membrane capacitance, $\mathrm{e}$ is electron charge, $k_{B}$ is Boltzmann's constant, and $\mathrm{T}$ is absolute temperature. $\mathrm{C}_{\mathrm{sa}}$ is a component of capacitance that characterizes sigmoidal changes in specific membrane capacitance ${ }^{12,26}$. Functional prestin density in the membrane is based on quantity of sensor charge.

$$
\begin{gathered}
Q_{\max }=\left[\frac{4 \cdot k_{B} \cdot T}{z \cdot e}\right] \cdot N L C_{V h} \\
\text { Prestin density }=\frac{Q_{\max }}{\text { patch surface area }}
\end{gathered}
$$

A power fit of NLC across frequency $(f)$ was performed ${ }^{13,27}$.

$$
N L C(f)=N L C_{0}+a * f^{b}
$$

where $\mathrm{NLC}_{0}$ is the zero frequency component, and $a$ and $b$ control the frequency response.

\section{Results}

In our present study, we successfully recorded from 25 macro-patches on the OHC lateral membrane, where membrane breakdown did not occur; breakdown might be expected beyond our voltage protocol voltages ${ }^{28}$. Our pipette inner tip diameter was $3.45+/-0.15 \mu \mathrm{m}$. $\mathrm{R}_{\mathrm{s}}$ of pipettes (under slight positive pressure) in bath prior to patching, determined by step voltages, was $1.34+/-0.04 \mathrm{M} \Omega$. However, this may not reflect series resistance following patch configuration. We therefore calculated $R_{s}$ given a typical resistivity for pipette solutions of 100 $\mathrm{ohm}-\mathrm{cm}^{29}$, and our pipette taper angle of $0.2 \mathrm{rad}$; we calculate an $\mathrm{R}_{\mathrm{s}}$ of about $920 \mathrm{k} \Omega$. Since the patch membrane extends into the pipette where pipette diameter is larger, we further estimate a reduction of $R_{s}$ to $735 \mathrm{k} \Omega$. The difference in NLC frequency response provided between measured and calculated $\mathrm{R}_{\mathrm{s}}$ were minimal, showing 
$\mathrm{a}-2.2 \mathrm{~dB}$ reduction in NLC at $30 \mathrm{kHz}$ for the directly measured values. Below we utilize the calculated value response.

The lateral membrane of the $\mathrm{OHC}$ is virtually devoid of voltage-dependent conductances ${ }^{11}$, and we additionally use channel blockers to insure this. Our seal resistance, determined by linear fit of step induced currents within a linear region of the I-V between -40 and $+40 \mathrm{mV}$, was $5.39+/-0.65 \mathrm{G} \Omega$.

Consequently, the macro-patch membrane, unlike the membrane in whole cell conditions, may be considered an isolated capacitor under voltage clamp, and thus amenable to determination of complex capacitance. In the following analysis of complex capacitance, we follow the methodology of Fernandez et al ${ }^{30}$, applied to each patch individually, based on its characteristics. The $\mathrm{OHC}$ patch capacitance presents as a parallel combination of linear $\left(\mathrm{C}_{\text {lin }}\right)$ and prestin-generated (NLC) capacitance. Conceivably, prestin's voltage-sensor may work as an imperfect, lossy capacitor that possesses both resistive (due to dielectric loss) and capacitive components (e.g., modelled as a combination of capacitor and resistor); that imperfection may influence estimates of $\mathrm{NLC}^{18}$. In addition to biophysical capacitance, system-generated stray capacitance $\left(\mathrm{C}_{\text {stray }}\right)$ will contribute to our measures. $\mathrm{C}_{\text {stray }}$ though voltage-independent, may also possess resistive and capacitive components, its admittance being $Y_{\text {stray }}^{*}(\omega)$. Under voltage clamp, an AC voltage across the patch membrane $\left(V_{m}\right)$ induces an $A C$ current $\left(I_{m}\right)$, where the $\operatorname{admittance}\left(Y_{m}=\mathrm{I}_{\mathrm{m}} / \mathrm{V}_{\mathrm{m}}\right)$ is a complex function of angular frequency, $\omega=2 \pi f$ and $i=\sqrt{-1}$

$$
Y_{m}(\omega)=G_{m}(\omega)+i B_{m}(\omega)
$$

with $G_{m}$ representing membrane conductance, $B_{m}$ representing membrane susceptance. Before continuing, we remove the effects of series resistance $\left(R_{s}\right)$ by subtracting it from the real component of membrane impedance $Z_{m}(\omega)\left(1 / Y_{m}(\omega)\right)$, and then converting back to admittance ${ }^{30} . Y_{m}(\omega)$ can be described in more detail,

$$
Y_{m}(\omega)=G_{m}(\omega)+G_{\text {leak }}+Y_{\text {stray }}^{*}(\omega)+i \omega C_{m}(\omega)
$$

where $\mathrm{C}_{\mathrm{m}}(\omega)=\mathrm{C}_{\text {lin }}+$ NLC.

$G_{\text {leak }}$ represents a DC leakage conductance. $C_{\text {lin }}$ is taken as frequency independent and via small signal analysis we seek to determine the frequency dependence of NLC, after removing $Y_{\text {stray }}^{*}(\omega)$. To our benefit, admittance at $+160 \mathrm{mV}$ lacks NLC ${ }^{12}$

$$
Y_{m}^{160}(\omega)=G_{m}(\omega)+G_{\text {leak }}+Y_{\text {stray }}^{*}(\omega)+i \omega C_{\text {lin }}
$$

Thus, subtraction of membrane admittance at $+160 \mathrm{mV}$ from those corresponding measures at all other holding potentials provides a differential admittance, $Y_{m}^{*}(\omega)$, devoid of stray capacitance effects.

$$
Y_{m}^{*}(\omega)=Y_{m}(\omega)-Y_{m}^{160}(\omega)=G_{m}^{*}(\omega)+i B_{m}^{*}(\omega)
$$

Actually, after subtraction, there remains a small differential residual nonlinear, voltage-dependent DC leakage conductance $\left(G_{\text {leak }}\right)$, which we remove from $G_{m}^{*}(\omega)$ by subtraction of the real part of $Y_{m}^{*}(\omega)$ at zero frequency. We determine this value to subtract by extrapolating to zero frequency with a linear fit of $\operatorname{Re}\left(Y_{m}^{*}(\omega)\right)$ between 24.41 and $463.86 \mathrm{~Hz}$ at each of the stepped holding potentials. This is akin to removing leakage conductance determined by prior DC step estimates, but has the further advantage of being determined during the actual chirp stimulation period.

Complex membrane capacitance, a function of angular frequency, is defined as (see Fernandez et al. ${ }^{30}$ )

$$
C_{m}^{*}(\omega)=\frac{Y_{m}^{*}(\omega)}{i \omega}=\frac{B_{m}^{*}(\omega)}{\omega}-i \frac{G_{m}^{*}(\omega)}{\omega}=C_{m}^{* \prime}(\omega)-i C_{m}^{*^{\prime \prime}}(\omega)
$$

In Fig. 1A, we plot the average capacitive (real, $\left.C_{m}^{* \prime}(\omega)\right)$ and apparent conductance (imaginary, $C_{m}^{* \prime \prime}(\omega)$ ) components of the complex capacitance for holding potentials of $40,0,-40$ and $-80 \mathrm{mV}$. The values are voltage dependent. While the capacitive component is large and frequency dependent, the conductance component is smaller and less frequency dependent, differing from a larger component predicted from diffusion-based charge translocation $^{18}$.

The absolute magnitude of the complex capacitance can be used to glean an estimate of membrane capacitance $\left(C_{m}^{1}(\omega)\right)$,

$$
C_{m}^{1}(\omega)=\left|C_{m}^{*}(\omega)\right|
$$

In Fig. 1B, the absolute magnitude, $\left|C_{m}^{*}(\omega)\right|$, of the complex capacitance is plotted. The lower panel plots the phase angle. The magnitude rolls off continuously across frequency at each holding potential. In Fig. 1C, the data are plotted as a specific complex capacitance, i.e., per linear capacitance of the patches. As found in whole cell recordings, peak NLC can be larger than linear capacitance ${ }^{5,6}$.

It is also possible to estimate capacitance of the macro-patch membrane by a "phase tracking" approach. That is, the phase angle of the complex admittance $Y_{m}^{*}(\omega)$ can be rotated to minimize its real component $\left(G_{m}^{*}(\omega)\right)$, providing a new value $\left(\left(Y_{m}^{* *}\right)\right)$, whose imaginary component reflects the capacitive component (susceptance) of the admittance in the absence of conductance interference. Another estimate of membrane capacitance, $C_{m}^{2}(\omega)$, can then be obtained. 

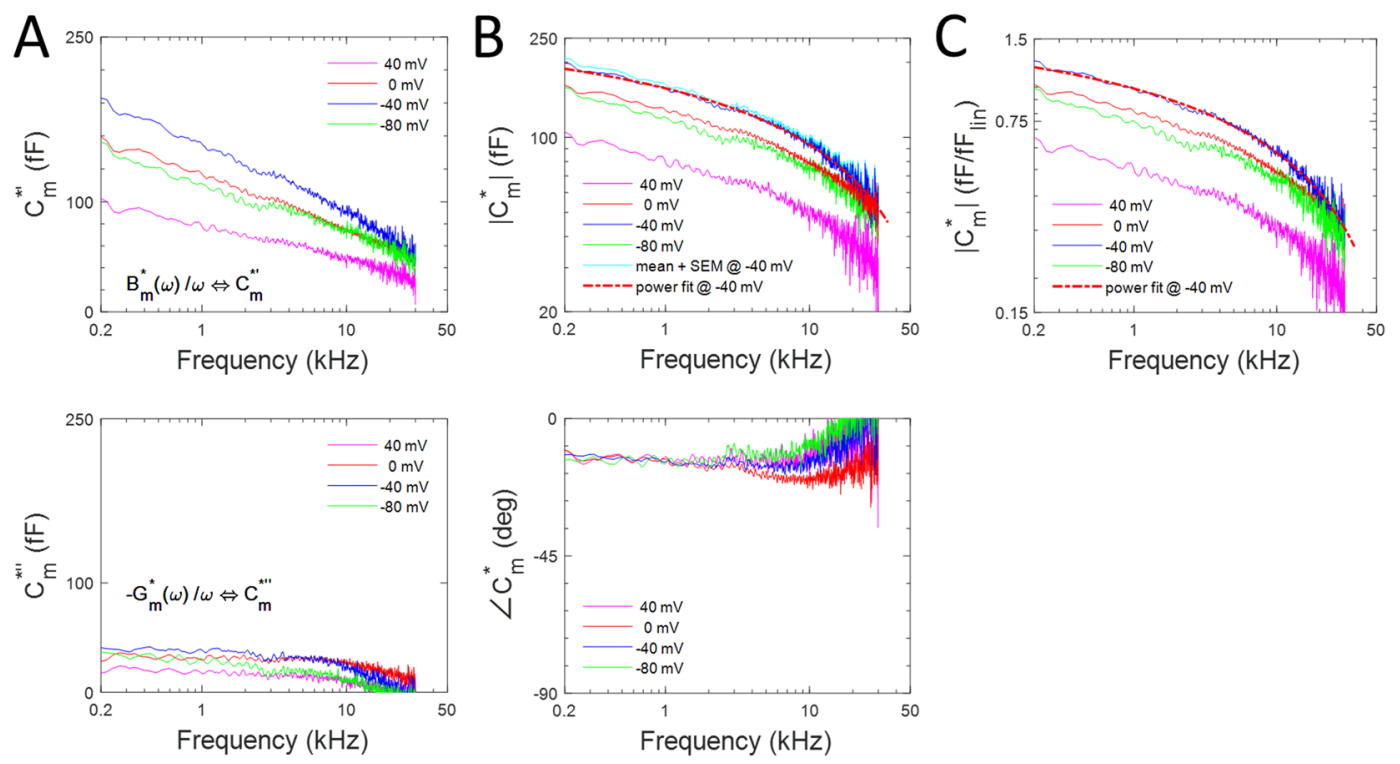

Figure 1. Complex capacitance of OHC lateral membrane macro-patches. (A) Top panel shows the mean real components of complex capacitance at selected holding potentials; bottom panel shows corresponding imaginary parts. (B) Top panel plots mean magnitudes of complex capacitance at different holding potentials. A power function fit of the magnitude at the $-40 \mathrm{mV}$ holding potential is shown by the dotted line (red) overlying the mean (dark blue line). The light blue line is the mean + SEM at that same holding potential data. Fit parameters: $\mathrm{NLC}_{0}: 279.17 \mathrm{fF}$, a: -34.35 , b: 0.18 . Bottom panel show the phase of complex capacitance. (C) Plot of the means of complex capacitance magnitude per estimated linear patch capacitance (see Methods). Fit parameters: $\mathrm{NLC}_{0}: 1.71 \mathrm{fF}, \mathrm{a}:-0.19$, b: 0.19 . Note low-pass behavior of magnitude functions.

$$
\begin{gathered}
\angle\left(Y_{m}^{*}(\omega)\right)=\operatorname{atan}\left(\frac{B_{m}^{*}(\omega)}{G_{m}^{*}(\omega)}\right) \\
Y_{m}^{* *}=Y_{m}^{*}(\omega) \cdot e^{\left(-i\left(\angle\left(Y_{m}^{*}(\omega)\right)-\frac{\pi}{2}\right)\right.} \\
C_{m}^{2}(\omega)=\frac{\operatorname{Im}\left(Y_{m}^{* *}\right)}{\omega}
\end{gathered}
$$

This approach is similar to traditional real time capacitance phase tracking under voltage clamp ${ }^{31}$, where the capacitive component at the angle of $\delta \mathrm{Y} / \delta \mathrm{C}_{\mathrm{m}}{ }^{32}$ is obtained by adjusting the lock-in recording angle until the conductance component is minimized and the capacitive component is maximized. In that approach, calibration with a known capacitance provides membrane capacitance estimates at the measurement frequency. This approach to measure OHC patch NLC was used by Gale and Ashmore ${ }^{14}$.

In Fig. 2A, we plot the two estimates of $\mathrm{OHC} \mathrm{NLC}\left[C_{m}^{1}(\omega)\right.$, and $\left.C_{m}^{2}(\omega)\right]$, corresponding to methods utilizing the complex capacitance magnitude, and the phase tracking approach, respectively - both at $-40 \mathrm{mV}$ holding potential $\left(\right.$ near $V_{h}$ ). The measures overlap, indicating that Eqs. 6 and 9 return the same result. In Fig. $2 \mathrm{~B}$, the voltage dependence of the complex capacitance magnitude at selected frequencies, presents a bell-shaped function typical of OHC NLC ${ }^{5,6}$, whose peak precipitously decreases with frequency, but whose voltage at peak $\left(\mathrm{V}_{\mathrm{h}}\right)$ remains similar across frequency. These data are fit (Eq. $\mathrm{m} 1)$ to extract the Boltzmann parameters given in the legend. Prestin density based on fits to capacitance at $1 \mathrm{kHz}$ is $1133 / \mu \mathrm{m}^{2}$, similar to previous estimates at a similar frequency ${ }^{8-10}$.

Having arrived at practical approaches to estimate OHC patch NLC, we now look at the influence of membrane tension. Several studies under whole cell voltage clamp have found that as membrane tension is increased, NLC $V_{h}$ shifts in the depolarizing direction, and decreases in peak magnitude ${ }^{15-17}$. However, utilizing lock-in estimates of lateral membrane patch capacitance instead of the whole cell technique, Gale and Ashmore ${ }^{9}$ found no evidence for a reduction in peak capacitance despite shifts in the depolarizing direction.

In Fig. 3, we explore the effects of membrane tension on the magnitude, $\left|C_{m}^{*}(\omega)\right|$, of complex NLC across frequency, as it is a robust measure of NLC, equivalent to that obtained with the phase tracking approach. Results at $0,-4,-8$ and $-10 \mathrm{~mm} \mathrm{Hg}$ (i.e., $0,0.53,1.06$ and $1.33 \mathrm{kPa}$ ) pipette pressure are shown in Fig. 3A-D, left panels. Membrane tension shifts $V_{h}$, as indicated by the rearrangement of the capacitance magnitude traces as negative pressure alters. For example, in left panel A, the trace at $-80 \mathrm{mV}$ (green) is above the trace at $0 \mathrm{mV}$ (red), whereas in left panel $\mathrm{D}$, the positions are reversed. In the right panels, plots of $\mathrm{C}-\mathrm{V}$ functions of complex capacitance magnitude at the four different pipette pressures are shown (mean $+/-$ SEM, $n=8$ ). The depolarizing shift in $V_{h}$ is 

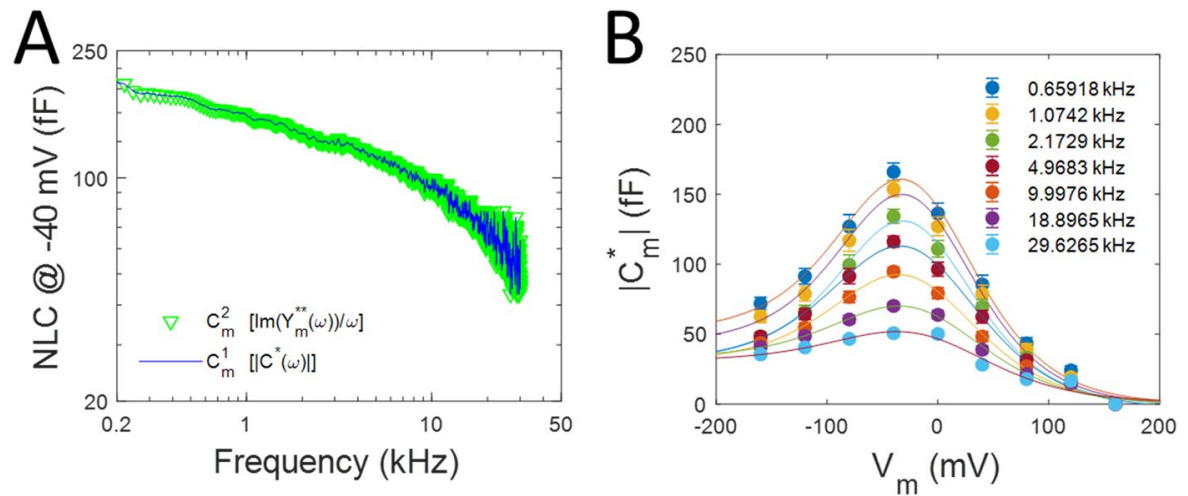

Figure 2. Comparison of two estimates of NLC frequency response at $-40 \mathrm{mV}$ holding potential. (A) Each estimate provides equivalent results. (B) $\mathrm{C}-\mathrm{V}$ plot of complex capacitance magnitude of OHC lateral membrane macro-patches at selected frequencies (mean $+/-$ SEM). Data are fit (Eq. $\mathrm{m} 1$ ) to extract Boltzmann parameters (see Methods). $-40 \mathrm{mV}$ is close to $\mathrm{V}_{\mathrm{h}}$. From lowest to highest frequency fit values are (peak NLC, $\mathrm{V}_{\mathrm{h}}, \mathrm{z}$ ): 133.60, $127.61,115.42,98.81,76.81,52.60,34.35 \mathrm{fF} ;-25.2,-25.3,-26.0,-26.4,-26.9,-20.6,-16.4 \mathrm{mV} ; 0.61,0.62$, $0.62,0.57,0.59,0.56,0.54$. Prior to averaging patch responses $(n=25)$, capacitance values from each patch were obtained by averaging over a bandwidth of about $200 \mathrm{~Hz}$ about the listed frequency value.

readily apparent as negative pressure increases (red bars). There is little change in peak capacitance, however. $\Delta \mathrm{C}_{\mathrm{sa}}$ is mainly unaffected by tension.

For comparison of frequency dependence as a function of membrane tension, Fig. 4A replots the peak magnitude traces $(-40 \mathrm{mV})$ from Fig. 3 at the different pipette pressures. The frequency response of complex capacitance magnitude is unaffected by membrane tension, indicated by the overlap of traces. The sensitivity of patch $\mathrm{V}_{\mathrm{h}}$ shift to tension was $24.1 \mathrm{mV} / \mathrm{kPa}$ based on our fits (Fig. $4 \mathrm{~B}$ ).

We previously measured OHC NLC in macro-patches using a dual-sinusoidal methodology ${ }^{13}$. In Fig. 5, we replot selected $\mathrm{NLC}_{\mathrm{Vh}}$ data points from that study (yellow circles) alongside $\mathrm{NLC}_{\mathrm{Vh}}$ data (green circles) from Gale and Ashmore ${ }^{14}$. We also plot whole cell data on $\mathrm{NLC}_{\mathrm{Vh}}$ (rat, mouse and guinea pig) from previous studies that used either the phase-tracking approach or 2-sine methodology. These scaled whole cell data interrogated a frequency bandwidth extending to $5 \mathrm{kHz}$, and closely follow the attenuation of patch NLC within that bandwidth. The Gale and Ashmore data (green circles, SEM, and fit from their Fig. 3B; in green) are also scaled (X 3.5) to overlie our data. Their measures were made with a lock-in amplifier using a $100 \mathrm{fF}$ calibration at each frequency. Scaling to our data was necessary because our average patch size/capacitance was greater than theirs. As is evident, our previous data intersects their data points, indicating an acceptable correspondence. Finally, extraction of $\mathrm{NLC}_{\mathrm{Vh}}$ from our new measures (red circles) of patch absolute complex capacitance (from Fig. 3, scaled X 1.05) corresponds well to our previous observations and theirs. We emphasize that the absolute magnitude of complex capacitance ${ }_{(} C_{m}^{1}(\omega)$ is equivalent to our phase tracking approach $C_{m}^{2}(\omega)$ ) to estimate capacitance (Fig. 2), each corresponding to the estimates provided by the traditional phase tracking method ${ }^{33}$ employed by Gale and Ashmore ${ }^{14}$. In the discussion, despite their assertions, we detail how Gale and Ashmore's data points above their $60 \mathrm{kHz}$ amplifier cut-off are actually valid data points depicting $\mathrm{NLC}_{\mathrm{Vh}}$. Capitalizing on the validity of their high frequency data, we combine all three patch data sets in the plot to arrive at a unified power function fit $\left(\mathrm{r}^{2}=0.97\right)$ of $\mathrm{NLC}_{\mathrm{Vh}}$ across frequency (solid magenta line), which shows precipitous roll-off of sensor charge movement relative to voltage drive. Fitted parameters are $\mathrm{NLC}_{0}: 185.2 \mathrm{fF}, \mathrm{a}:-7.22$, and b: 0.28 . At $77 \mathrm{kHz}, \mathrm{NLC}_{\mathrm{Vh}}$ is $40 \mathrm{~dB}$ down from $\mathrm{NLC}_{0}$. Furthermore, since each method takes into account the dielectric loss in prestin, we conclude that this dielectric loss does not significantly account for the low-pass nature of prestin charge movement. To further substantiate this claim, we make another estimate of membrane capacitance, $C_{m}^{3}(\omega)$, one that does not take into account dielectric loss, but is appropriate for a loss-less capacitor.

$$
C_{m}^{3}(\omega)=\frac{\operatorname{Im}\left(Y_{m}^{*}(\omega)\right)}{\omega}=\frac{B_{m}^{*}(\omega)}{\omega}
$$

Figure 6 shows the results for our three estimates of prestin capacitance $\left[C_{m}^{1}(\omega), C_{m}^{2}(\omega)\right.$ and $\left.C_{m}^{3}(\omega)\right]$, all reasonably overlying each other, and exhibiting low-pass behavior. Thus, all results available to date show an unusually low-pass behavior of prestin voltage-sensor performance, with little influence of dielectric loss.

\section{Discussion}

We previously evaluated OHC macro-patch NLC frequency dependence utilizing a dual-sine methodology that worked on extracted prestin displacement currents ${ }^{13}$. That approach did not allow separation of real and imaginary components of NLC. Here we provide an analysis of OHC NLC in membrane macro-patches utilizing single sine methods that have been used to characterize gating charge movements in other voltage-dependent proteins $\mathrm{s}^{30}$, where real (capacitive) and imaginary ("resistive", representing dielectric loss) components of sensor charge are separated. We explored these alternative approaches in order to determine whether new estimates of NLC frequency response incorporating the influence of dielectric loss would substantially alter our previous estimates, 

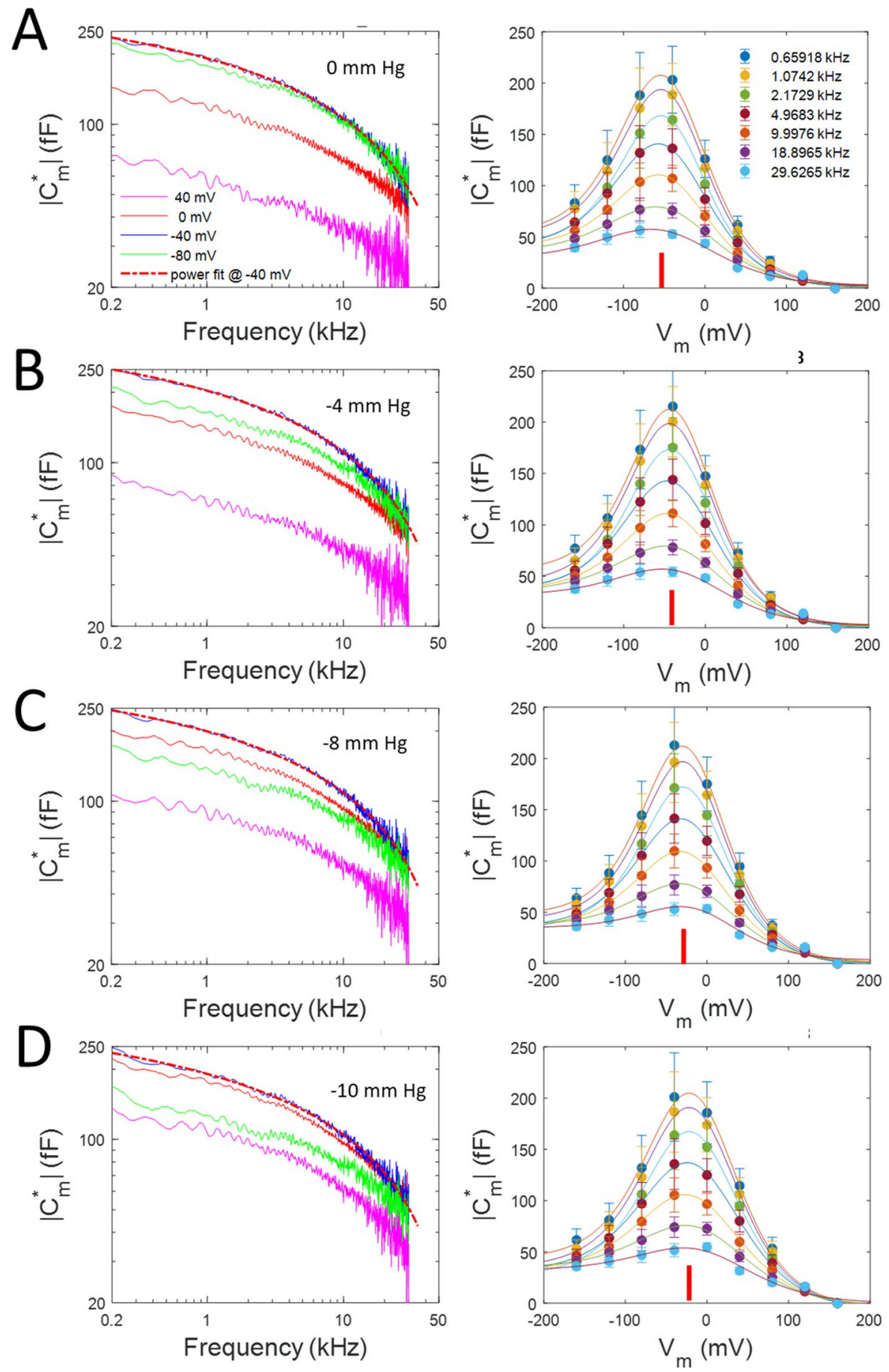

Figure 3. Effect of membrane tension on magnitude of complex capacitance voltage and frequency dependence. Panels (A-D) depict results at $0,-4,-8$ and $-10 \mathrm{~mm} \mathrm{Hg}$ pipette pressure. The effect of membrane tension is to shift $V_{h}$, as indicated by the change in voltage at maximal capacitance as negative pressure increases, without appreciably altering frequency response (dotted lines are fits to a power function). On the right, $\mathrm{C}-\mathrm{V}$ plots of complex capacitance magnitude at the four different pipette pressures are shown. Red bars indicate voltage at peak capacitance. The depolarizing shift in $\mathrm{V}_{\mathrm{h}}$ is readily apparent as pressure increases. Peak magnitudes do not appreciably alter. $\Delta \mathrm{C}_{\mathrm{sa}}$ is tension and frequency independent. Mean $+/-\operatorname{SEM}(\mathrm{n}=8)$. From lowest to highest frequency fit values from Eq. $\mathrm{m} 1$ are (peak NLC, $\mathrm{V}_{\mathrm{h}}, \mathrm{z}$ ): Panel A, 179.15, 166.45, 146.43, $120.30,88.90,62.62,41.51 \mathrm{fF} ;-49.4,-49.0,-49.0,-50.9,-49.0,-48.2,-51.2 \mathrm{mV} ; 0.70,0.71,0.71,0.66,0.70$, 0.58, 0.53; Panel B, 182.80 175.62, 153.91, 124.40, 91.56, 60.38, 39.08 fF; -38.5, -40.0, -39.4, -40.7, -39.4, $-36.4,35.8 \mathrm{mV} ; 0.74,0.72,0.72,0.67,0.66,0.60,0.57$. Panel C, $187.73,175.16,155.35,124.08,90.68,57.97,34.77$ $\mathrm{fF} ;-25.9,-25.7,-25.7,-26.2,-26.0,-20.6,-14.5 \mathrm{mV} ; 0.73,0.73,0.72,0.67,0.66,0.62,0.65$. Panel D, 185.03, $175.67,156.00,124.51,91.22,58.39,36.32$, fF; $-17.2,-17.7,-18.0,-18.1,-8.8,-12.3,-8.0 \mathrm{mV} ; 0.67,0.66$, $0.66,0.61,0.60,0.58,0.55$. 

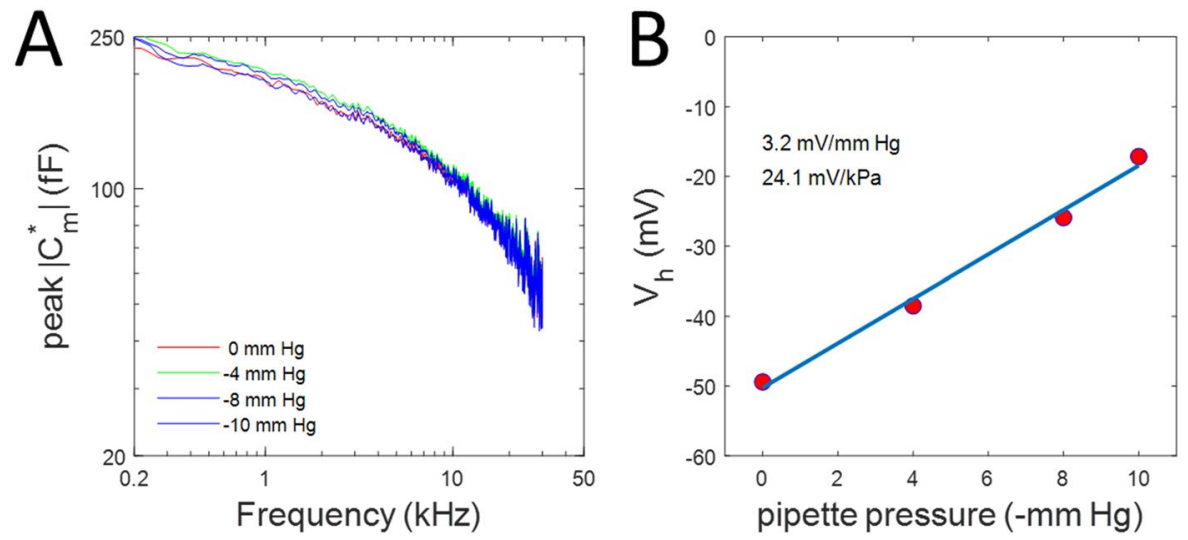

Figure 4. Effects of membrane tension on NLC frequency response and operating voltage. (A) Comparison of NLC frequency response at $-40 \mathrm{mV}$ at four pipette pressures. Roll-off is equivalent as indicated by overlap of traces. (B) $\mathrm{V}_{\mathrm{h}}$ of NLC at the lowest frequency in Fig. 3 is plotted versus pipette pressure. A linear fit (blue line) indicates a sensitivity of $24.1 \mathrm{mV} / \mathrm{kPa}$.

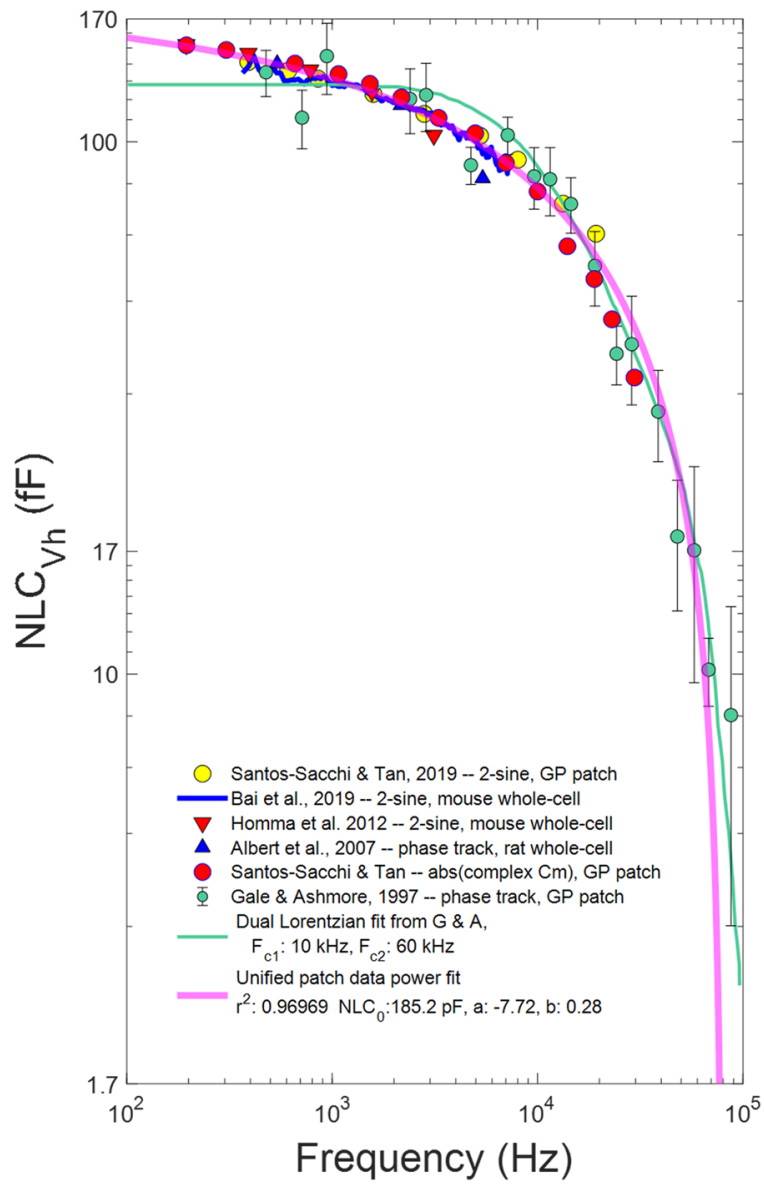

Figure 5. Replot of some whole-cell NLC data ${ }^{27,45,46}$, and NLC patch data from Santos-Sacchi and Tan ${ }^{13}$ with those of Gale and Ashmore ${ }^{14}$. Using a dual-sine capacitance estimation algorithm, NLC of OHC patches (yellow circles) overlie the data of Gale and Ashmore, collected with a traditional phase tracking approach using a lock-in amplifier (data: green circles; their fit green solid line). Whole cell data, both 2 -sine and phase-tracking, show a similar frequency response within their interrogation bandwidth. Finally, $\mathrm{NLC}_{\mathrm{Vh}}$ (red symbols) obtained from Eq. $\mathrm{ml}$ fits of our complex capacitance data (obtained as in Fig. 2B) are also commensurate with previous observations, giving a unified power fit for the patch data (magenta line; see Discussion) with parameters $\mathrm{NLC}_{0}$ : $185.2 \mathrm{fF}, \mathrm{a}:-7.22$, b: 0.28 . 


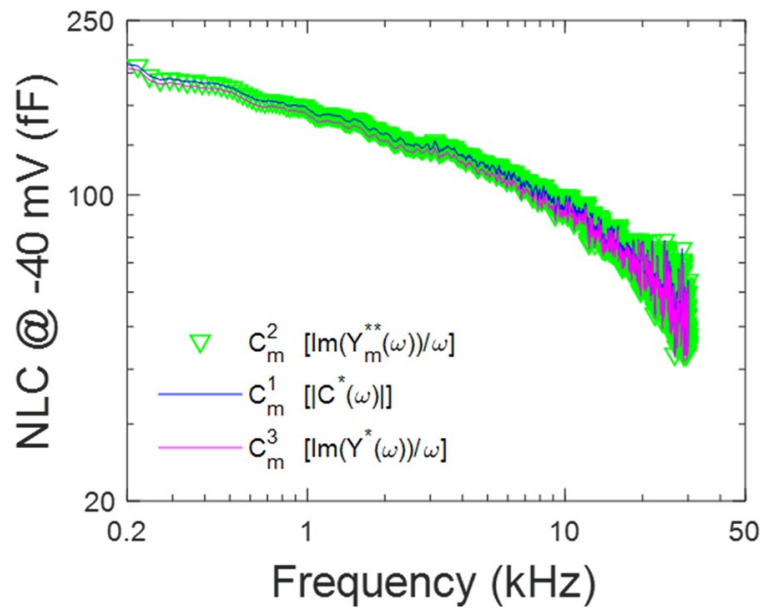

Figure 6. Three estimates of the frequency response of NLC at $-40 \mathrm{mV}$ holding potential. Estimates from raw admittance data (pink line) reasonably compare with the other estimates despite the fact that the real component is not taken into account.

as was predicted through modelling ${ }^{18}$. This is not the case, however. Thus, the bulk of prestin charge movement appears to move in phase with voltage, and the small dielectric loss that we find does not significantly influence measures of OHC NLC frequency response.

It was previously noted that low frequency determined OHC NLC $(\mathrm{C}-\mathrm{V})$ measures are unable to identify whether prestin charge movement is multistate/diffusional in nature ${ }^{8,34}$. However, wide-band frequency interrogation of prestin NLC may provide clues. In this regard, the large bell-shaped (across frequency) resistive component of complex capacitance, which is associated with $\mathrm{DPA}^{-}$voltage-driven translocation through bulk membrane lipid ${ }^{35}$, and which Sun et al. ${ }^{18}$ predicted for prestin modelled as a simple diffusion process of charge translocation is not found here. The resistive component we measure is relatively small and flat across frequency (Fig. 1A, lower panel). This is likely because prestin charge movement is not a diffusional process, but instead similar to gating charge movement in other membrane proteins. Thus, Fernandez et al. ${ }^{36}$ found that whereas chloroform, which alters membrane viscosity, can influence the speed of DPA ${ }^{-}$charge translocation within the membrane, $\mathrm{Na}$ channel voltage-sensor charge translocation speed is not affected. Similarly, sensor charge in prestin is likely constrained to charged protein residues that move during protein conformational change ${ }^{37}$. Indeed, Sun et al's model included membrane diffusion of chloride ions as voltage sensors in prestin. However, chloride anions do not solely function as extrinsic voltage sensors for prestin, nor do they move through bulk lipid; instead they likely influence prestin kinetics ${ }^{13,38,39}$ (also see our comment \#1 on Walter et al. eLife 8, 2019 - https://elifesciences.org/articles/46986) ${ }^{40}$.

Prestin's voltage operating range, whose midpoint is at $V_{h}$, is very sensitive to membrane tension ${ }^{15}$. Here, we measured the effect of membrane tension on prestin performance by changing patch pipette pressure to study its influence on NLC frequency response. The average sensitivity of patch $\mathrm{V}_{\mathrm{h}}$ shift to tension was $24.1 \mathrm{mV} / \mathrm{kPa}$, which corresponds well to our previous measures ${ }^{17}$ in whole cell mode of $21.6 \mathrm{mV} / \mathrm{kPa}$. Adachi et al. ${ }^{41}$ found similar sensitivity in whole cell mode $(27.5 \mathrm{mV} / \mathrm{kPa})$. Gale and Ashmore ${ }^{9}$, in their patches, found a somewhat smaller sensitivity of $11.1 \mathrm{mV} / \mathrm{kPa}$. In prestin transfected cells, values of near $4 \mathrm{mV} / \mathrm{kPa}$ were found ${ }^{42,43}$. One mechanism whereby shifts in $\mathrm{V}_{\mathrm{h}}$ might occur would be due to changes in the ratio of transition rates between its conformational states. Naturally, if this were occurring then changes in NLC frequency response might arise. We found no change in NLC magnitude, similar to results of Gale and Ashmore ${ }^{9}$, or frequency response during alterations in membrane tension, and conclude that tension is not altering transition rates. Indeed, we previously found that membrane tension in whole cell mode did not significantly alter the phase or frequency response of electromotility $^{44}$. This is in contrast to salicylate which likely alters transition rates since NLC frequency response is affected ${ }^{13}$.

Lastly, we revisited the seminal patch data of Gale and Ashmore ${ }^{14}$, along with those whole-cell NLC measures of others ${ }^{27,45,46}$, and recast our current and previous patch data ${ }^{13}$ with those prior results on OHC NLC. The small variability within our current and previous patch NLC data set allowed us to identify a power dependence of frequency, whereas Gale and Ashmore originally applied a Lorentzian fit. Their interpretation of NLC frequency response requires re-examination. They asserted that their capacitance measures made above their amplifier's $60 \mathrm{kHz}$ cut-off frequency were inaccurate, being unduly reduced by this cut-off. However, the amplifier's transfer function effect on the phase tracking approach ${ }^{24,31,33}$ is inconsequential, since the lock-in phase is adjusted to maximize the imaginary (capacitive) component and minimize the real (resistive) component of the recorded signal. That is, the phase shift due to the amplifier was eliminated, and amplifier attenuation was corrected by their $100 \mathrm{pF}$ calibration. Thus, their small-signal capacitance measurement was truly that of the patch membrane (and possibly non-voltage dependent stray capacitance), but when fitted by a Boltzmann derivative across voltage, provided valid, not invalid, estimates of voltage-dependent OHC NLC above the amplifier cut-off. Consequently, their fit to OHC NLC data at $V_{h}$, which included the amplifier roll-off component, actually provided a dual-Lorentzian evaluation of OHC NLC, and their conclusion that only the lower frequency Lorentzian component of the fit 
fully characterized the behavior of OHC NLC (10 kHz cut-off) was misplaced. Furthermore, their interpretation suggested that OHC NLC roll-off is $6 \mathrm{~dB}$ /octave, when, in fact, roll-off is more than double that at high frequencies and is actually frequency dependent. Finally, the proposed inaccuracies in their high frequency estimates provided leeway to dismiss low-pass NLC indications of electromechanical behavior in favor of eM measures that were flat beyond $80 \mathrm{kHz}^{47}$, the latter driving our concept of OHC eM's wideband influence on cochlear amplification over the last couple of decades. In addition to all of our frequency evaluations of OHC NLC, past ${ }^{13,20,23,48,49}$ and present, recent eM measures, in vitro and in vivo, have challenged that concept ${ }^{20,44,48,50}$.

In summary, Fig. 5 shows that a power function fit to all patch data to-date reasonably describes the roll-off in $\mathrm{NLC}_{\mathrm{Vh}}$. This unifying observation highlights an inability of voltage to sufficiently drive prestin electromechanical activity at very high frequencies $(60-160 \mathrm{kHz})$, where CA (cochlear amplification) is known or expected to exist. The additional low pass influence of external loads on the whole cell eM frequency response and the frequency-dependent RC membrane time constant that we characterized recently ${ }^{48}$, also lessens the effectiveness of voltage-dependent eM in influencing organ of Corti motion at very high frequencies. This point is further emphasized by experiments showing that perilymphatic perfusion of $5 \mathrm{mM}$ salicylate or its congener is devastating to $\mathrm{CA}^{51,52}$. Considering the $1.6 \mathrm{mM} \mathrm{K}_{1 / 2}$ of salicylate action, the effect of the perilymphatic treatment is not a full block of NLC, but rather about $75 \%(12 \mathrm{~dB})$ reduction ${ }^{53}$. Thus, the reduction in NLC resulting from its power frequency dependence ( $12 \mathrm{~dB}$ down at $25 \mathrm{kHz}, 20 \mathrm{~dB}$ down at $53 \mathrm{kHz}$, and $40 \mathrm{~dB}$ down at $77 \mathrm{kHz}$; Fig. 5) likely has biological impact across the full auditory frequency spectrum of mammals, and indicates a continuously diminishing influence of voltage alone to drive prestin electromechanical activity and CA via a cycle-by-cycle means. As a caveat, it may be possible that at any given frequency the measured NLC/eM amplitude is sufficient to drive CA, as we implied previously ${ }^{20,54}$, and that a reduction in CA that might occur with salicylate could be working on the existent magnitude at that particular frequency.

Two additional issues need to be addressed. They are temperature dependence, and species dependence of NLC frequency response. It is likely that temperature dependence of NLC frequency response is not so great $\left(\mathrm{Q}_{10}\right.$ of 1.33), as Gale and Ashmore (1997) have directly attested to in membrane patches. We had previously argued that because $\mathrm{V}_{\mathrm{h}}$ is highly temperature sensitive ${ }^{55,56}$, the kinetics of prestin should be, as well - we estimated a $Q_{10}$ of 2 based on modelling of $V_{h}$ shifts observed in IR temperature jump experiments ${ }^{57}$. But, since we now find that membrane tension does not influence prestin kinetics, in spite of shifts in $V_{h}$, it is possible that temperature-induced shifts, likewise, work not through kinetics, but by some other means. Given that one-half magnitude NLC is at $7 \mathrm{kHz}$ (from the power fit, Fig. 5), a $\mathrm{Q}_{10}$ of 1.33 would increase the cut-off to only about $10 \mathrm{kHz}$. Concerning species specificity, the data so far do not support such differences, since rat, mouse and guinea pig show similar frequency responses (see Fig. 5). Whether genetic variations in prestin that have been observed in mammals that capitalize on very high frequency detection ${ }^{58,59}$ could change this view remains possible, and we need to test these species. Currently, however, how CA is driven by OHCs in the basal high frequency turn of mammals that enjoy perception of sound above $60 \mathrm{kHz}$ is an enigma.

Received: 6 February 2020; Accepted: 26 March 2020;

Published online: 10 April 2020

\section{References}

1. Zheng, J. et al. Prestin is the motor protein of cochlear outer hair cells. Nature 405, 149-155 (2000).

2. Santos-Sacchi, J. \& Dilger, J. P. Whole cell currents and mechanical responses of isolated outer hair cells. Hear. Res. 35, 143-150 (1988).

3. Kachar, B., Brownell, W. E., Altschuler, R. \& Fex, J. Electrokinetic shape changes of cochlear outer hair cells. Nature 322, 365-368 (1986).

4. Liberman, M. C. et al. Prestin is required for electromotility of the outer hair cell and for the cochlear amplifier. Nature 419, 300-304 (2002).

5. Santos-Sacchi, J. Reversible inhibition of voltage-dependent outer hair cell motility and capacitance. J. Neurosci. 11, 3096-3110 (1991).

6. Ashmore, J. F. Forward and reverse transduction in the mammalian cochlea. Neurosci. Res. Suppl. 12, S39-S50 (1990).

7. Santos-Sacchi, J. Fast outer hair cell motility: how fast is fast? in The Mechanics and Biophysics of Hearing (eds Dallos, P. et al.) 69-75 (Springer-Verlag, 1990).

8. Huang, G. \& Santos-Sacchi, J. Mapping the distribution of the outer hair cell motility voltage sensor by electrical amputation. Biophys. J. 65, 2228-2236 (1993).

9. Gale, J. E. \& Ashmore, J. F. The outer hair cell motor in membrane patches. Pflugers Arch. 434, 267-271 (1997).

10. Mahendrasingam, S., Beurg, M., Fettiplace, R. \& Hackney, C. M. The ultrastructural distribution of prestin in outer hair cells: a postembedding immunogold investigation of low-frequency and high-frequency regions of the rat cochlea. Eur. J. Neurosci. 31, 1595-1605, doi:EJN7182 [pii];10.1111/j.1460-9568.2010.07182.x [doi] (2010).

11. Santos-Sacchi, J., Huang, G. J. \& Wu, M. Mapping the distribution of outer hair cell voltage-dependent conductances by electrical amputation. Biophys. J. 73, 1424-1429 (1997).

12. Santos-Sacchi, J. \& Navarrete, E. Voltage-dependent changes in specific membrane capacitance caused by prestin, the outer hair cell lateral membrane motor. Pflugers Arch. 444, 99-106 (2002).

13. Santos-Sacchi, J. \& Tan, W. Voltage Does Not Drive Prestin (SLC26a5) Electro-Mechanical Activity at High Frequencies Where Cochlear Amplification Is Best. iScience 22, 392-399, https://doi.org/10.1016/j.isci.2019.11.036 (2019).

14. Gale, J. E. \& Ashmore, J. F. An intrinsic frequency limit to the cochlear amplifier. Nature 389, 63-66 (1997).

15. Iwasa, K. H. Effect of stress on the membrane capacitance of the auditory outer hair cell. Biophys. J. 65, 492-498 (1993).

16. Gale, J. E. \& Ashmore, J. F. Charge displacement induced by rapid stretch in the basolateral membrane of the guinea-pig outer hair cell. Proc. R. Soc. Lond. B. Biol. Sci. 255, 243-249 (1994).

17. Kakehata, S. \& Santos-Sacchi, J. Membrane tension directly shifts voltage dependence of outer hair cell motility and associated gating charge. Biophys. J. 68, 2190-2197 (1995).

18. Sun, S. X. et al. Voltage and frequency dependence of prestin-associated charge transfer. J. Theor. Biol. 260, 137-144, doi:S0022-5193(09)00231-8 [pii];10.1016/j.jtbi.2009.05.019 [doi] (2009).

19. Ratnanather, J., Brownell, W. \& Popel, A. Mechanical properties of the outer hair cell. Biophysics of hair cell sensory systems, 199-206 (1993). 
20. Santos-Sacchi, J. \& Tan, W. The Frequency Response of Outer Hair Cell Voltage-Dependent Motility Is Limited by Kinetics of Prestin. J. Neurosci. 38, 5495-5506, https://doi.org/10.1523/JNEUROSCI.0425-18.2018 (2018).

21. Wu, M. \& Santos-Sacchi, J. Effects of lipophilic ions on outer hair cell membrane capacitance and motility. J.Membr.Biol. 166, 111-118 (1998).

22. Dallos, P., Evans, B. N. \& Hallworth, R. Nature of the motor element in electrokinetic shape changes of cochlear outer hair cells. Nature 350, 155-157 (1991).

23. Santos-Sacchi, J. \& Song, L. Chloride anions regulate kinetics but not voltage-sensor Qmax of the solute carrier SLC26a5. Biophys. J. 110, 1-11 (2016)

24. Gillis, K. D. Techniques for Membrane Capacitance Measurements In Single Channel Recording (eds. Sakmann, B. \& Neher, E.) Ch. 7, 155-198 (Plenum Press, 1995).

25. Hille, B. Ionic channels of excitable membranes. 2nd edn, (Sinauer Associates, 1992).

26. Santos-Sacchi, J. \& Song, L. Chloride and Salicylate Influence Prestin-Dependent Specific Membrane Capacitance: Support for the Area Motor Model. J Biol Chem, https://doi.org/10.1074/jbc.M114.549329 (2014).

27. Bai, J. P., Navaratnam, D. \& Santos-Sacchi, J. Prestin kinetics and corresponding frequency dependence augment during early development of the outer hair cell within the mouse organ of Corti. Sci. Rep 9, 16460, https://doi.org/10.1038/s41598-019-52965-1 (2019).

28. Navarrete, E. G. \& Santos-Sacchi, J. On the effect of prestin on the electrical breakdown of cell membranes. Biophys. J. 90, 967-974 (2006).

29. Mathias, R. T., Cohen, I. S. \& Oliva, C. Limitations of the whole cell patch clamp technique in the control of intracellular concentrations. Biophys. J. 58, 759-770, https://doi.org/10.1016/S0006-3495(90)82418-8 (1990).

30. Fernandez, J. M., Bezanilla, F. \& Taylor, R. E. Distribution and kinetics of membrane dielectric polarization. II. Frequency domain studies of gating currents. J.Gen.Physiol 79, 41-67 (1982).

31. Fidler, N. \& Fernandez, J. M. Phase tracking: an improved phase detection technique for cell membrane capacitance measurements. Biophys. J. 56, 1153-1162, https://doi.org/10.1016/S0006-3495(89)82762-6 (1989).

32. Santos-Sacchi, J. Determination of cell capacitance using the exact empirical solution of $\mathrm{dY} / \mathrm{dCm}$ and its phase angle. Biophys. J. 87, 714-727 (2004)

33. Neher, E. \& Marty, A. Discrete changes of cell membrane capacitance observed under conditions of enhanced secretion in bovine adrenal chromaffin cells. Proc. Natl. Acad. Sci. USA. 79, 6712-6716 (1982).

34. Scherer, M. P. \& Gummer, A. W. How many states can the motor molecule, prestin, assume in an electric field? Biophys. J. (2005).

35. Lu, C. C. et al. Membrane transport mechanisms probed by capacitance measurements with megahertz voltage clamp. Proc. Natl. Acad. Sci. USA. 92, 11220-11224 (1995)

36. Fernandez, J. M., Bezanilla, F. \& Taylor, R. E. Effect of chloroform on charge movement in the nerve membrane. Nature 297, 150-152, https://doi.org/10.1038/297150a0 (1982)

37. Bai, J. P. et al. Prestin's anion transport and voltage-sensing capabilities are independent. Biophys. J. 96, 3179-3186, doi:S0006-3495(09)00470-6 [pii];10.1016/j.bpj.2008.12.3948 [doi] (2009).

38. Song, L. \& Santos-Sacchi, J. Conformational state-dependent anion binding in prestin: evidence for allosteric modulation. Biophys. J. 98, 371-376, doi:S0006-3495(09)01669-5 [pii];10.1016/j.bpj.2009.10.027 [doi] (2010).

39. Rybalchenko, V. \& Santos-Sacchi, J. Allosteric modulation of the outer hair cell motor protein prestin by chloride. In Biophysics of the Cochlea: From Molecules to Models (ed. Gummer, A.) 116-126 (World Scientific Publishing, 2003).

40. Walter, J. D., Sawicka, M. \& Dutzler, R. Cryo-EM structures and functional characterization of murine Slc26a9 reveal mechanism of uncoupled chloride transport. Elife 8, https://doi.org/10.7554/eLife.46986 (2019).

41. Adachi, M., Sugawara, M. \& Iwasa, K. H. Effect of turgor pressure on outer hair cell motility. J.Acoust. Soc. Am. 108, 2299-2306 (2000).

42. Ludwig, J. et al. Reciprocal electromechanical properties of rat prestin: The motor molecule from rat outer hair cells. Proc. Natl. Acad. Sci. U. S. A. 98, 4178-4183 (2001).

43. Santos-Sacchi, J., Shen, W., Zheng, J. \& Dallos, P. Effects of membrane potential and tension on prestin, the outer hair cell lateral membrane motor protein. J. Physiol 531, 661-666 (2001)

44. Santos-Sacchi, J. \& Song, L. Chloride-driven Electromechanical Phase Lags at Acoustic Frequencies Are Generated by SLC26a5, the Outer Hair Cell Motor Protein. Biophys. J. 107, 126-133, https://doi.org/10.1016/j.bpj.2014.05.018 (2014).

45. Homma, K., Duan, C., Zheng, J., Cheatham, M. A. \& Dallos, P. The V499G/Y501H mutation impairs fast motor kinetics of prestin and has significance for defining functional independence of individual prestin subunits. J Biol Chem 288, 2452-2463, https://doi. org/10.1074/jbc.M112.411579 (2013).

46. Albert, J. T. et al. Voltage-sensitive prestin orthologue expressed in zebrafish hair cells. J. Physiol 580, 451-461 (2007).

47. Frank, G., Hemmert, W. \& Gummer, A. W. Limiting dynamics of high-frequency electromechanical transduction of outer hair cells. Proc. Natl. Acad. Sci. U. S. A. 96, 4420-4425 (1999).

48. Santos-Sacchi, J., Iwasa, K. H. \& Tan, W. Outer hair cell electromotility is low-pass filtered relative to the molecular conformational changes that produce nonlinear capacitance. J. Gen. Physiol. 151, 1369-1385, https://doi.org/10.1085/jgp.201812280 (2019).

49. Santos-Sacchi, J. High frequency measures of OHC nonlinear capacitance (NLC) and their significance: Why measures stray away from predictions. AIP Conference Proceedings 1965, 060004-060001-060004-060005, https://doi.org/10.1063/1.5038477 (2018).

50. Vavakou, A., Cooper, N. P. \& van der Heijden, M. The frequency limit of outer hair cell motility measured in vivo. Elife 8, https://doi. org/10.7554/eLife.47667 (2019).

51. Santos-Sacchi, J., Song, L., Zheng, J. F. \& Nuttall, A. L. Control of mammalian cochlear amplification by chloride anions. J. Neurosci. 26, 3992-3998, https://doi.org/10.1523/jneurosci.4548-05.2006 (2006).

52. Fisher, J. A., Nin, F., Reichenbach, T., Uthaiah, R. C. \& Hudspeth, A. J. The spatial pattern of cochlear amplification. Neuron 76, 989-997, doi:S0896-6273(12)00885-9 [pii];10.1016/j.neuron.2012.09.031 [doi] (2012).

53. Kakehata, S. \& Santos-Sacchi, J. Effects of salicylate and lanthanides on outer hair cell motility and associated gating charge. J. Neurosci. 16, 4881-4889 (1996).

54. Santos-Sacchi, J. The speed limit of outer hair cell electromechanical activity. HNO 67, 159-164, https://doi.org/10.1007/s00106019-0615-9 (2019).

55. Santos-Sacchi, J., Meltzer, J. \& Folkinshteyn, D. Temperature dependence of prestin's nonlinear capacitance. Society for Neuroscience Abstracts 27, 1053-1053 (2001)

56. Santos-Sacchi, J. \& Huang, G. Temperature dependence of outer hair cell nonlinear capacitance. Hear. Res. 116, 99-106 (1998).

57. Okunade, O. \& Santos-Sacchi, J. IR laser-induced perturbations of the voltage-dependent solute carrier protein SLC26a5. Biophys. J. 105, 1822-1828, https://doi.org/10.1016/j.bpj.2013.09.008 (2013).

58. Liu, Z., Qi, F. Y., Zhou, X., Ren, H. Q. \& Shi, P. Parallel sites implicate functional convergence of the hearing gene prestin among echolocating mammals. Mol. Biol. Evol. 31, 2415-2424, https://doi.org/10.1093/molbev/msu194 (2014).

59. Davies, K. T., Cotton, J. A., Kirwan, J. D., Teeling, E. C. \& Rossiter, S. J. Parallel signatures of sequence evolution among hearing genes in echolocating mammals: an emerging model of genetic convergence. Heredity (Edinb.) 108, 480-489, doi:hdy2011119 [pii];10.1038/hdy.2011.119 [doi] (2012). 


\section{Acknowledgements}

This research was supported by NIH-NIDCD R01 DC000273, R01 DC016318 and R01 DC008130 to J.S.-S. We thank Fred Sigworth for confirming our conclusions that the phase tracking approach to capacitance measurement is insensitive to patch clamp amplifier roll-off.

\section{Author contributions}

J.S.S. wrote the paper, designed and performed experiments and analyzed data. W.T. edited manuscript, performed experiments and analyzed data.

\section{Competing interests}

The authors declare no competing interests.

\section{Additional information}

Correspondence and requests for materials should be addressed to J.S.-S.

Reprints and permissions information is available at www.nature.com/reprints.

Publisher's note Springer Nature remains neutral with regard to jurisdictional claims in published maps and institutional affiliations.

(c) (i) Open Access This article is licensed under a Creative Commons Attribution 4.0 International License, which permits use, sharing, adaptation, distribution and reproduction in any medium or format, as long as you give appropriate credit to the original author(s) and the source, provide a link to the Creative Commons license, and indicate if changes were made. The images or other third party material in this article are included in the article's Creative Commons license, unless indicated otherwise in a credit line to the material. If material is not included in the article's Creative Commons license and your intended use is not permitted by statutory regulation or exceeds the permitted use, you will need to obtain permission directly from the copyright holder. To view a copy of this license, visit http://creativecommons.org/licenses/by/4.0/.

(c) The Author(s) 2020 